\title{
A strategic Ser/Cys exchange in the catalytic triad unlocks an acyltransferase mediated synthesis of thioesters and tertiary amides
}

Martina L. Contente ${ }^{1,2}$, David Roura-Padrosa², Francesco Molinari*3, Francesca Paradisi*1,2

${ }^{1}$ School of Chemistry, University of Nottingham, University Park, Nottingham, NG7 2RD, United Kingdom.

${ }^{2}$ Department of Chemistry and Biochemistry, University of Bern, Freiestrasse 3, 3012 Bern, Switzerland.

${ }^{3}$ Department of Food, Environmental and Nutritional Sciences (DeFENS), University of Milan, via Mangiagalli 25, 20133 Milan, Italy.

*Corresponding authors: Francesco Molinari, e-mail: francesco.molinari@unimi.it, tel: +390250319148; Francesca Paradisi, e-mail: francesca.paradisi@dcb.unibe.ch, tel: $+41(0) 316314002$

\section{Abstract}

Expanding the toolbox of enzymatic reactions accessible to organic chemists is one of the major goals in biocatalysis. Here, we describe the development of an acyltransferase variant from Mycobacterium smegmatis (MsAcT) where a strategic Ser/Cys exchange in the catalytic triad dramatically expanded its synthetic capability, yielding a biocatalyst able to efficiently catalyse the formation of thioesters and tertiary amides in water. Preparative scale (250 mM) biotransformations were performed starting from different thiols and secondary amines with excellent yields and reactions times, using vinyl esters as acylating agents. The high substrate-tocatalyst ratio and the cofactor independence make this process a sustainable and cost-effective procedure that was successfully applied to the synthesis of acetyl-CoA as well as structurally simpler analogues. Computational studies provided insights into enzymatic selectivity and substrate recognition.

The enzyme toolbox available for synthetic chemistry dramatically expanded in the last few years thanks to new high-throughput screening techniques and protein engineering. ${ }^{1,2}$ Enzymatic reactions can be nowadays used in preparative chemistry, enabling new easy, mild, and high yielding methods. Nevertheless, a few key-reactions are noticeably missing from this toolbox especially in terms of productivity and biocatalyst availability, such as thioester and tertiary amide preparation; proof of concepts have been reported, but no suitable preparative biocatalytic 
methodology is currently available. Both moieties are useful building blocks for organic chemistry, in particular thioesters play an important role as reactive donors in acyl transfer reaction ${ }^{3}$ while the amide-bond is a core linkage in many pharmaceuticals. ${ }^{4}$ Traditionally, they are prepared by condensation between thiols or secondary amines and carboxylic acids in the presence of activating agents. ${ }^{5,6,7}$ However, the starting material (e.g., acyl chloride), needs to be prepared insitu as it is generally moisture sensitive, limiting the practicality of this approach. Typical syntheses are performed in organic solvents requiring dry environments to avoid hydrolytic reactions. In particular, reported procedures for amide preparation are not considered environmentally friendly, and the manufacturing of these compounds is deemed a green chemistry challenge. ${ }^{8}$ In the enzyme panorama, lipases play a dominant role in condensation reactions in organic media, albeit scarce examples are reported for the thioester and tertiary amide synthesis. ${ }^{9}$ TL IM lipase from Thermomyces lanuginosus and D-amino acylase from E. coli have been applied for the preparation of thioesters in organic solvent, but low space time yields (STY), turnover number (TON) and limited substrate range were observed despite the use of high amount of catalyst. ${ }^{10,11}$ Similarly, Candida antarctica lipase B (CALB) was employed to prepare an intermediate tertiary amide towards the synthesis of alfuzosin, but again with an extremely poor catalyst-to-substrate ratio. $^{12}$

While the enzymatic synthesis of secondary amides counts many examples, ${ }^{4}$ that of tertiary amides is rarely observed. Aside from CALB, ATP-dependent carboxylic acid reductases (CARs) were also tested for the preparation of a tertiary amide of pharmaceutical relevance. Despite the large excess of secondary amine employed (100 eq.), the product was obtained with low yield (19\%) due to poor enzyme stability. ${ }^{13}$ Finally, a screening of $67 \mathrm{~N}$-acyltransferases resulted in only one able to synthesise tertiary amides with low-to-modest yields (5-41\%). ${ }^{14}$

Recently, we reported on an acyltransferase from Mycobacterium smegmatis (MsAcT) ${ }^{15,16}$ able to efficiently catalyse ester and amide formation in water both in batch $^{8,17}$ and under flow conditions. ${ }^{18,19}$ Wild-type (WT) MsAcT preferentially accepts primary alcohols and amines, although few selected secondary alcohols have also been acylated; ${ }^{20}$ Finnveden et al. developed MsAcT variants to further expand the $O$-acyl donor specificity. ${ }^{21}$ MsAcT shares common features with SGNH superfamily of serine-hydrolases, including the catalytic triad (Ser-His-Asp) ${ }^{22}$ that is evolutionary well conserved. Specifically, in the GDSL-like family which includes MsAcT as well as hydrolytic enzymes (e.g, lipases and peptidases), both catalytic serine and histidine are extremely conserved (>99\% of the analysed sequences) (See Supplementary Figure 2). In the typical 
mechanism of acyltransferases, the catalytic triad leads to the formation of the acyl-enzyme intermediate (acyl-O-Ser-enzyme) which may not be sufficiently reactive for softer or bulkier nucleophiles. Considering the synthetic MsAcT capability in water, engineering this enzyme to generate a higher energy acyl-enzyme even more susceptible to nucleophilic attacks, could lead to a different set of acylated molecules. In such scenario, the mutation of the catalytic Ser for a Cys will generate a more reactive acyl-S-Cys-enzyme, favouring the Cys-S- elimination step not only by $\mathrm{H}$-bonding in the oxyanion hole, but also by poor $p$-orbital overlap between the $S$-atom and the carbonyl group. However, such Ser/Cys exchange in the catalytic triad of serine-hydrolases significantly reduced their activity, $23,24,25,26$ unless additional engineering is carried out to rescue their hydrolytic ability. ${ }^{27}$ Interestingly such mutation has never been explored in serine-containing acyltransferases and synthetic applications.

Here, we propose the Ser/Cys exchange as single rational mutation in the catalytic pocket of MsAcT to expand its synthetic capability in aqueous medium. The S11C mutant demonstrated a broader substrate scope towards thiols as well as secondary amines, which are not accepted by the WT. High activity towards alcohols was retained, making the developed enzyme extremely versatile. The access to valuable thioesters and tertiary amides in a greener and affordable way includes an efficient strategy to prepare one of the most important molecules in Nature, acetyl coenzyme $A$ and its analogues.

\section{Results}

\section{Synthesis of thioesters.}

The activity of S11C MsAcT mutant was assayed using 2-phenylethanethiol (1a) as model substrate to probe the influence of the nucleophilic exchange Ser/Cys. Vinyl acetate and isopropenyl acetate $(10 \% \mathrm{v} / \mathrm{v})$ were used as acetyl donors and the formation of S-phenethyl ethanethioate (2a) was obtained with excellent conversion (>99\%), and reaction times (30 min and $5 \mathrm{~h}$, respectively). Hydrolytic side-reactions, typical of prolonged batch transesterification with WT MsAcT, were never observed, in agreement with the reduced hydrolytic activity of mutated hydrolases mentioned earlier. S-acylation mediated by S11C MsAcT was further established by probing its substrate scope: a range of thiols on a $250 \mathrm{mM}$ scale were tested with vinyl acetate as donor to demonstrate such syntheses beyond the analytical scale (Table 1). 
All thiols gave the corresponding thioesters with quantitative or nearly quantitative conversion (81->99\%) and rapid reaction times (0.5-3 h). High substrate loading (250 mM, 23-35 g/L) and catalyst concentration of $1 \mathrm{mg} / \mathrm{mL}(0.04 \mathrm{mM})$ highlight the preparative potential of the evolved enzyme. Unlike the WT, S11C MsAcT does not accept ethyl acetate as acyl donor, as it is not sufficiently activated. The enzyme tolerance towards different vinyl ester donors was further investigated with two selected thiols $(\mathbf{1} \mathbf{a} ; \mathbf{1} \mathbf{b})$ (Table 2$)$.

S-acylation occurred with excellent molar conversion (70->99\%) and relatively short reaction times (2-6 h) also with branched vinyl esters (e.g., vinyl methacrylate).

While the Cys-mutation successfully showed that thioesters are now easily accessible, the real leap in its application is the synthesis of more relevant products such as acetyl coenzyme A (acetylCoA) and its mimetics for which, to date, there is no straightforward preparation.

Acetyl-CoA can be enzymatically accessed via acetyl-CoA (ATP-dependent) synthetase, and plays an essential role in many biological pathways (e.g., fatty acids, esters, polyketides, non-ribosomal peptides). ${ }^{28,29}$ In addition, acetyl-CoA is often a key reagent in tailored in-vitro/in-vivo studies, and strategies for its preparation and in-situ regeneration would be extremely useful as the cost of CoA-thioesters is often prohibitive.

In ex-vivo biosynthesis, the truncated CoA analogue, $N$-acetylcysteamine (HS-NAC, Figure 1 ), has recently found widespread use, as its $S$-acetyl derivative mimics CoA-thioesters in most cases. ${ }^{30}$

$\mathrm{N}$-(2-mercaptoethyl)acetamide 10 (HS-NAC, $250 \mathrm{mM}$ ) was quickly transformed in its S-acetylderivative 20 with quantitative conversion (Figure 1). Such reaction was carried out on a $10 \mathrm{~mL}$ scale affording $380 \mathrm{mg}$ of pure compound ( $94 \%$ isolated yield). In addition, S11C MsAcT accepts $N$ Boc derivative 1p with identical performance. Finally, the $S$-acetylation of CoA, a significantly more complex molecule, was selectively achieved. No reaction with adenosine diphosphate (ADP) was observed even at enzyme concentrations of $5 \mathrm{mg} / \mathrm{mL}$ after $72 \mathrm{~h}$ (See Supplementary Methods). Previous attempts to prepare acyl-CoA derivatives relied almost exclusively on chemical ligation. These approaches however, suffer from several drawbacks including poor yields and low selectivity. ${ }^{31}$ Moreover, CoA and its conjugates are known for their instability, especially in organic solvents. ${ }^{30}$ Despite the discovery of various acyl-CoA synthetases, enzymatic reactions are limited 
by the high substrate specificity of the available biocatalysts. ${ }^{30} \mathrm{~A}$ chemoenzymatic synthesis of different acyl-CoA scaffolds was reported, but the $S$-acetylation step was still performed chemically. ${ }^{31}$ The cofactor independent S11C MsAcT efficiently transformed large amounts of CoA (100 $\mathrm{mM}$ ) to acetyl-CoA (80\%) employing simply vinyl acetate as donor. As described above, the absence of hydrolytic activity allows the enzyme to work for $48 \mathrm{~h}$ without the formation of sideproducts. In addition, the synthesised acetyl-CoA and analogue $\mathbf{2 0}$ were successfully used as crude preparations in a two-step NADH-dependent in-vitro assay demonstrating their efficiency as reagents in acetyl-CoA-dependent enzymatic cascades (See Supplementary Methods).

While CoA is expensive, we have shown here how the developed enzyme can replace CoA synthetase and could be employed in more complex reactions mimicking ex-vivo cellular metabolism where acetyl-CoA regeneration is needed.

\section{Computational studies}

To gain insight on the binding of acetyl-CoA and its mimetics, docking studies revealed that while 20 could be oriented both with the $N$ - and $S$-acetyl group for the catalysis to take place, with the hindered $\mathbf{2 p}$ only the $S$-acetyl group could fit in the active site (See Supplementary Figure 4). Acetyl-CoA, on the other hand, displayed the best score of all the tested molecules $(-7.8 \mathrm{kcal} / \mathrm{mol})$ when the thioester carbonyl oxygen points towards the oxyanion hole and in a catalytic distance from Cys11 (Figure 2).

To confirm the results obtained with docking algorithms, ${ }^{32}$ molecular mechanics combined with generalised Born and surface area continuum solvation analysis of the substrate binding through MMPBSA.py included in AmberTools, ${ }^{33}$ were used. For the tested $\mathbf{1 0}$ and acetyl-CoA, the complex substrate-active site was simulated for 2 ns. In both cases, the substrates adopted a catalytically active conformation (See Supplementary Figure 6), showing very similar, favourable, binding energies (See Supplementary Table 2). ${ }^{34}$.

Previous reports on WT MsAcT identified a tunnel leading to the buried active site with a strong hydrophobic character. ${ }^{15,22}$ To better understand how a complex, bulky hydrophilic molecule as CoA could be accepted as a substrate, in silico docking and transport analysis were performed.

In addition to the highly hydrophobic channel involving 3 sub-units of the octamer $(26.4 \AA), 15,22$ using Caver 3 software a second larger tunnel (29.6 $)$ involving the central part of the protein was 
identified, effectively lengthening the previously suggested channel (See Figure 3 and Supplementary Figure 7).

The larger tunnel presents a bottleneck (1.25 $\AA$ ) with a marked polar nature formed by residues from two chains (loop 63-67 and T116). To study the CoA transport to the active site, as well as the acetyl-CoA exit in the opposite direction, CaverDock software was employed. ${ }^{35,36}$ An energy profile of the substrate in its path to the catalytic pocket (Figure 3), as well as the product on the reverse direction, was obtained. Interestingly, CoA tends to approach the catalytic pocket presenting the adenine first. Eventually, a flip of the structure is observed positioning the thiol in a catalytically accepted conformation with respect to Cys11 (See Supplementary Figure 8 and Supplementary video).

\section{Synthesis of tertiary amides}

It was quickly discovered that the enhanced performance of S11C MsAcT was not limited to soft thiol nucleophiles. WT MsAcT showed excellent activity with primary amines, ${ }^{8,18}$ but secondary amines, which would lead to highly valuable tertiary amides, were not accepted (See Supplementary Methods). Here, a range of secondary and cyclic amines (250 mM) were tested using vinyl acetate as donor (Table 3).

Tertiary amides were obtained with good results (30->99\%), in reasonable reaction times (2-24 h) demonstrating the versatility of S11C MsAcT. 3d (entry 4, Table 3) was the only secondary amine not accepted. Here, this simple yet strategic single point mutation expanded the substrate scope of the cofactor-independent MsAcT beyond expectations, obtaining a stable and easy-to-handle enzyme able to perform condensation reactions on a preparative scale in water where the amine is the limiting reagent, and simple mildly activated esters are used in moderate excess ( $\sim$ eq.).

\section{Stereoselectivity of MsAcT S11C}

WT MsAcT exhibits only moderate enantiopreference with selected substrates. ${ }^{37}$ MsAcT S11C was therefore assessed with branched nucleophiles (entries 1-7, Table 4) and an $\alpha$-substituted cyclic secondary amine (entries 8-10, Table 4) using vinyl acetate as donor. Low-to-moderate $(R)$ enantiopreference was observed for compounds $\mathbf{5 a}$ and $\mathbf{5 b}$, while only $(R)$-1-phenylethanol (5f) was accepted as substrate in the acetylation reaction. Interestingly, when the racemic $\mathbf{5 e}$ was tested, no reaction was observed suggesting that the $(S)$-enantiomer may have an inhibiting effect. 
Notably $\alpha$-methylbenzylamines (entries 2-4, Table 4), not accepted by the WT, ${ }^{38}$ are suitable substrates for MsAcT S11C, further expanding the substrate scope of this variant. The sterically hindered 2-phenylpyrrolidine (entry 8, Table 4) was converted with high S-selectivity (6 h, 25\% m.c., $>99 \%$ e.e.). Computationally, we observed that the $(R)$-enantiomer (entry 10, Table 4), when forced in a catalytically active conformation, presents clashes with lysine 97 and loops near the active site preventing an efficient binding (See Supplementary Figure 5).

\section{Discussion}

It is known that the catalytic properties of most Ser-/Cys-hydrolases are remarkably different indicating that they evolved separately. ${ }^{27}$ Within this family, an example of Ser/Cys nucleophilic exchange which occurred in evolutionary terms regards proteases. While interconversion of the catalytic serine with cysteine is in principle possible, simple Ser/Cys mutation is often accompanied by notable reduction in hydrolytic activity. In contrast, this has never been studied in the transferase family sharing the same catalytic triad, and we supposed that could be converted into an advantage for designing an evolved enzyme with synthetic properties.

In our study, the single mutation Ser/Cys in the catalytic triad of MsAcT (Ser-His-Asp) unlocked the enzyme mediated synthesis of thioesters and hindered amides, giving access to a series of compounds which can now be sustainably prepared on preparative scale in aqueous media. The peculiar structural and catalytic features of MsAcT, which inherently limits its hydrolytic abilities in favour of its synthetic capacity in water, were further enhanced by the mutation. The mutant, tolerating high substrate loading and working without the addition of cofactors, makes the process very attractive and efficient in terms of STY and TON $\left(5-6 \times 10^{3}\right)$ which, for thioester synthesis, is four orders of magnitude higher than the mentioned enzymatic examples. ${ }^{11}$ The WT used as a control, never showed activity towards thiols and secondary amines even at high catalyst concentrations. Acetyl-CoA and its analogues were prepared with excellent conversions (80$>99 \%$ ), and very good reaction times. Notably, under our working conditions, no spontaneous reaction was observed in the absence of the catalyst. To verify their possible application in enzymatic assembly lines, in-vitro assays were performed on the synthesised, crude acetyl-CoA and structurally simplified analogues, demonstrating their suitability as starting material. Hydrolytic side reactions were never observed, thus enhancing the selective preparation of the desired product. The chemoselectivity of the developed protein, was investigated experimentally and computationally. While reactions involving ADP were carried out without any acetylation result, the COA catalytic orientation was demonstrated through docking and transport analysis. 
Moderate $(R)$-enantiopreference on branched nucleophiles was retained by the mutated enzyme, while the cyclic 2-phenylpyrrolidine was transformed with high e.e. for the S-enantiomer.

In summary, this work provides an example of the construction of an evolved enzyme able to transform structurally different substrates not accepted by the WT. This sustainable procedure can be considered a valid biocatalytic alternative for the preparation of two of the most important classes of molecules in Nature: amides and thioesters. The ex-vivo efficient and affordable biocatalytic preparation of acetyl-CoA and its analogues opens the path to the possibility to involve S11C MsAcT in more complex reactions with the final aim of mimicking a cellular metabolism.

\section{Methods}

\section{Materials}

All reagents and solvents were obtained from commercial suppliers and were used without further purification.

\section{Mutation, Overexpression and Purification of S11C MsAcT}

A single point mutation $\mathrm{S} 11 \mathrm{C}$ was performed using the Q5 site-directed mutagenesis kit (New England Biolabs) through the following primers: 5'-GGCTGGGTTCCGGTTGAAG-3' as forward and 3'GAAGCCGCTAAcaGACTGGACC-5' as reverse. The resulting plasmid pET26b-S11C-MsAcT was transformed into chemically competent E. coli BL21star(DE3) for heterologous expression. Culture of $E$. coli BL21star(DE3)-pET26b-S11C-MsAcT (20 mL) was grown overnight at $37^{\circ} \mathrm{C}$ in LB medium supplemented with $25 \mu \mathrm{g} / \mathrm{mL}$ kanamycin. $200 \mathrm{~mL}$ of cultivation medium (Terrific Broth: $12 \mathrm{~g} / \mathrm{L}$ bacto-tryptone, $24 \mathrm{~g} / \mathrm{L}$ yeast extract, $4 \mathrm{~g} / \mathrm{L}$ glycerol, $2.3 \mathrm{~g} / \mathrm{L} \mathrm{KH}_{2} \mathrm{PO}_{4}, 9.4 \mathrm{~g} / \mathrm{L} \mathrm{K}_{2} \mathrm{HPO}_{4}, 25 \mu \mathrm{g} / \mathrm{mL}$ kanamycin, $\mathrm{pH} 7.2$ ) were inoculated with the previous described seed culture to an initial $\mathrm{OD}_{600 \mathrm{~nm}}$ of 0.1 . Cultivation was carried out at $37{ }^{\circ} \mathrm{C}, 150$ rpm. Cells were grown until $O D_{600 n m}$ reached the value of 0.5. The expression of S11C MsAcT was induced by the addition of isopropyl- $\beta$-D-thiogalactopyranoside (IPTG) to a final concentration of $0.1 \mathrm{mM}$. The culture was further incubated for $16 \mathrm{~h}$ at $25^{\circ} \mathrm{C}$. Cells were then harvested by centrifugation ( $20 \mathrm{~min}, 4500$ rpm, $4{ }^{\circ} \mathrm{C}$ ), washed once with $20 \mathrm{mM}$ phosphate buffer $\mathrm{pH} 8.0$ and stored at $-20{ }^{\circ} \mathrm{C}$.

$5 \mathrm{~g}$ of pellet were resuspended in $25 \mathrm{~mL}$ loading buffer $(100 \mathrm{mM}$ phosphate buffer $\mathrm{pH} 8.0,30 \mathrm{mM}$ imidazole, $100 \mathrm{mM} \mathrm{NaCl}$ ) and sonicated ( 5 cycles of $2 \mathrm{~min}$ each, in ice, with $1 \mathrm{~min}$ interval). Cell debris were harvested by centrifugation ( $45 \mathrm{~min}, 15000 \mathrm{rpm}, 4^{\circ} \mathrm{C}$ ). The enzyme was purified by affinity chromatography with HIS-Select ${ }^{\circledR}$ Nickel Affinity Gel. Briefly, the column was equilibrated with loading buffer and the crude extract loaded; the column was then washed with loading buffer; finally, the adsorbed enzyme was eluted with elution buffer (phosphate buffer $100 \mathrm{mM} \mathrm{pH} \mathrm{8.0,250} \mathrm{mM} \mathrm{imidazole,} 100 \mathrm{mM} \mathrm{NaCl}$ ).

Crude extract, pellet and pure protein were analysed by SDS-PAGE (See Supplementary Figure 9). The fractions showing the presence of a band of the expected size $(25.6 \mathrm{kDa})$ were pooled, dialyzed against 100 
$\mathrm{mM}$ phosphate buffer $\mathrm{pH} 8.0$ and stored at $4{ }^{\circ} \mathrm{C}$. Typically, starting from $5 \mathrm{~g}$ of wet cell paste, it was possible to obtain $100 \mathrm{mg}$ of pure protein $(5 \mathrm{mg} / \mathrm{mL})$.

\section{Small scale batch reactions}

Batch reactions using S11C MsAcT were performed in $10 \mathrm{~mL}$ screw cap tubes, in duplicates; $1 \mathrm{~mL}$ reaction mixture in $100 \mathrm{mM}$ phosphate buffer $\mathrm{pH} 8.0$, containing $250 \mathrm{mM}$ substrate (100 mM in the case of CoA), 1 $\mathrm{mg} / \mathrm{mL}$ enzyme, and $10 \% \mathrm{v} / \mathrm{v}$ acyl donor were left under magnetic stirring at $25{ }^{\circ} \mathrm{C}$. $50 \mu \mathrm{L}$ aliquots were collected at different reaction times. For CoA reactions, samples were opportunely diluted in the mobile phase and directly analysed by HPLC. For the other biotransformations they were extracted with $100 \mu \mathrm{L}$ of EtOAc. In the case of amine substrates before the extraction, samples were quenched with $\mathrm{NaOH} 1 \mathrm{M}$. The reactions were monitored by TLC (synthesis of thioesters: $n$-Hexane/EtOAC 8:2; synthesis of amides: $\mathrm{CH}_{2} \mathrm{Cl}_{2} / \mathrm{MeOH}$ 9:1 + 0.1\% TEA). Samples, after anhydrification were prepared for GC analysis or after evaporation, resuspended in the mobile phase for HPLC analysis (See Supplementary Methods).

\section{0-mL scale biotransformations}

Batch reactions using S11C MsAcT were performed in a 25-mL round-bottom flask, in duplicates; $10 \mathrm{~mL}$ reaction mixture in $100 \mathrm{mM}$ phosphate buffer $\mathrm{pH} 8.0$, containing $250 \mathrm{mM} 10$ or $1 \mathrm{p}, 1 \mathrm{mg} / \mathrm{mL}$ enzyme, and $10 \% \mathrm{v} / \mathrm{v}$ vinyl acetate were left under magnetic stirring at $25^{\circ} \mathrm{C}$. After $30 \mathrm{~min}$ the biotransformations were extracted with EtOAc $(5 \mathrm{~mL} \times 3)$. The crude was treated with $\mathrm{Na}_{2} \mathrm{SO}_{4}$ and the solvent removed under reduced pressure. $380 \mathrm{mg}$ of pure compound $\mathbf{2 0}$ (94\% yield) and $520 \mathrm{mg}$ of $\mathbf{2 p}$ (95\% yield) were obtained. After GC analysis (See Supplementary Methods), they were tested as reported above without any further purification.

\section{Assay of Acetyl-CoA and mimetics}

The formation and employment of Acetyl-CoA and analogues as starting material for more complex reactions was demonstrated through the "Determination of acetic acid Kit" ( $\mathrm{r}$-Biopharm). Samples were prepared and assayed following the kit instructions. Measurements were performed spectrophotometrically at $340 \mathrm{~nm}$ observing the formation of NADH (See Supplementary Methods), at 25 ${ }^{\circ} \mathrm{C}$ in a half-microcuvette at determined times.

\section{Data availability statement}

All data are available upon request directly to the corresponding authors. The crystal structure used as the starting point for the in silico analysis is available in the Protein Data Bank (https://www.rcsb.org/) under accession number 2Q0S. The dataset for conservation analysis can be found in https://pfam.xfam.org/family/PF13472.

\section{References}

1. Sheldon, R.A., Pereira, P.C. Biocatalysis engineering: the big picture. Chem. Soc. Rev. 46, 2678-2691 (2017).

2. Devine, P.N. et al. Extending the application of biocatalysis to meet the challenges of drug development. Nat. Rev. Chem. 2, 409-421 (2018). 
3. Jordan, A., Sneddon, H.F. Development of a solvent-reagent selection guide for the formation of thioesters. Green Chem. 21, 1900-1905 (2019).

4. Petchey, M.R., Grogan G. Enzyme-Catalysed Synthesis of Secondary and Tertiary Amides. Adv. Synth. Catal. 361, 3895-3914 (2019).

5. YI, C.L., Huang, Y.T., Lee, C.F. Synthesis of thioesters through copper catalyzed coupling of aldehydes with thiols in water. Green Chem. 15, 2476-2484 (2013).

6. Hirschbeck, V., Gehrtz, P.H., Fleischer, I. Metal-catalyzed synthesis and use of thioesters: recent developments. Chem. Eur. J. 24, 7092-7107 (2018).

7. Pattabiraman, R.V., Bode, J.W. Rethinking amide bond synthesis. Nature, 480, 471-479 (2011)

8. Contente, M.L., Pinto, A., Molinari, F., Paradisi, F. Biocatalytic N-Acylation of Amines in Water Using an Acyltransferase from Mycobacterium smegmatis. Adv. Synth. Catal. 360, 4814-4819 (2018).

9. Lustosa de Melo Carvalho, A.C. et al. Recent Advances in Lipase-Mediated Preparation of Pharmaceuticals and Their Intermediates. Int. J. Mol. Sci. 16, 29682-29716 (2015).

10. Lou, F.W., Liu, B.K., Wang, J.L. Pan, Q., Lin, X. F. Controllable enzymatic Markovnikov addition acylation of thiols to vinyl esters. J. Mol. Catal. B-Enzym. 60, 64-68 (2009).

11. Zhou, N. et al. Enzymatic synthesis of thioesters from thiols and vinyl esters in a continuous-flow microreactor. Catalysts, 8, 249-261 (2018).

12. Balessari, A., Mangone, C.P. One-pot biocatalyzed preparation of substituted amides as intermediates of pharmaceuticals. J. Mol. Catal. B : Enzym. 11, 335-341 (2001).

13. Wood, A.J.L. et al. Adenylation activity of carboxylic acid reductases enables the synthesis of amides. Angew. Chem. 129, 14690-14693 (2017).

14. Philpott, H.K., Thomas, P.J., Tew, D., Fuerst, D.E., Lovelock, S.L. A versatile approach to amide bond formation. Green Chem, 20, 3426-3431 (2018).

15. Mathews, I. et al. Structure of a novel enzyme that catalyzes acyl transfer to alcohols in aqueous conditions. Biochemistry 46, 8969-8979 (2007).

16. Wiermans, L. et al. Transesterifications and peracid-assisted oxidations in aqueous media catalyzed by Mycobacterium smegmatis acyl transferase. ChemCatChem 5, 3719-3724 (2013).

17. Perdomo, I. et al. Efficient Enzymatic Preparation of Flavor Esters in Water. J. Agr. Food Chem. 67, 6517-6522 (2019).

18. Contente, M.L., Farris, S., Tamborini, L., Molinari, F., Paradisi, F. Flow-based enzymatic synthesis of melatonin and other high value tryptamine derivatives: A five-minute intensified process. Green Chem. 21, 3263-3266 (2019).

19. Contente M.L., Tamborini, L., Molinari, F., Paradisi F. Aromas flow: eco-friendly, continuous, and scalable preparation of flavour esters. J. Flow Chem. 10, 235-240 (2020).

20. de Leeuw, N. et al. Ester Synthesisin Water: Mycobacterium smegmatis Acyl Transferase for Kinetic resolution. Adv. Synth. Catal. 360, 242-249 (2018).

21. Finnveden, M., Semlitsch, S., He, O., Martinelle, M. Mono-substitution of symmetric diesters: selectivity of Mycobacterium smegmatis acyltransferase variants. Catal. Sci. Technol. 9, 4920-4927 (2019).

22. Kazemi, M., Sheng, X., Kroutil, W., Himo, F. Computational Study of Mycobacterium smegmatis Acyl Transferase Reaction Mechanism and Specificity. ACS Catal. 8, 1069810706 (2018).

23. Neet, K.E., Koshland, D.E. Jr. The conversion of serine at the active site of subtilisin to cysteine: A “chemical mutation" Proc. Natl. Acad. Sci. USA 56, 1606-1611 (1966). 
24. Polgar, L., Bender, M.L. A new enzyme containing a synthetically formed active site Thiolsubtilisin. J. Am. Chem. Soc. 88, 3153-3154 (1966).

25. Higaki, J.N., Evnin, L.B., Craik, C.S. Introduction of a cysteine protease active site into trypsin. Biochemistry 28, 9256-9263 (1989).

26. Wilke, M., Higaki, J.N., Craik, C.S., Fletterick, R.J., Crystal structure of rat tripsin-S195C at $150{ }^{\circ} \mathrm{C}$ : Analysis of low activity of recombinant and semisynthetic thiol protease. J. Mol. Biol. 219, 511-523 (1991).

27. Cen, $Y$. et al. Artificial cysteine-lipases with high activity and altered catalystic mechanism created by laboratory evolution. Nat. Commun. 10, 1-10 (2019).

28. Staunton, J., Weissman, K. Polyketide biosynthesis: A millennium review. J. Nat. Prod. Rep. 18, 380-416 (2001).

29. Mercer, A.C., Burkart, M.D. The ubiquitous carrier protein-a window to metabolite biosynthesis. Nat. Prod. Rep. 24, 750-773 (2007).

30. Franke, J., Hertweck, C. Biomimetic Thioesters as Probes for Enzymatic Assembly Lines: Synthesis, Applications and Challeges. Cell Chem. Biol. 23, 1179-1192 (2016).

31. Agarwal, V. et al. Chemoenzymatic Synthesis of Acyl Coenzyme A Substrates Enables in Situ Labelling of Small Molecules and Proteins. Org. Lett. 17, 4452-4455 (2015).

32. Torres, P.H.M., Sodero, A.C.R., Jofily, P., Silva-Jr, F.P. Key Topics in Molecular Docking for Drug Design. Int. J. Mol. Sci. 20, 4574 (2019).

33. Miller, B.R. et al. MMPBSA.py: An Efficient Program for End-State Free Energy Calculations. J. Chem. Theory Comput. 8, 3314-3321 (2012).

34. Wang, E. et al. End-Point Binding Free Energy Calculation with MM/PBSA and MM/GBSA: Strategies and Applications in Drug Design. Chem. Rev. 119, 9478-9508 (2019).

35. Vávra, O. et al. CaverDock: a molecular docking-based tool to analyse ligand transport through protein tunnels and channels. Bioinformatics 35, 1-8 (2019).

36. Filipovic, J. et al. CaverDock: A Novel Method for the Fast Analysis of Ligand Transport. IEEE/ACM Trans. Comput. Biol. Bioinf. doi: 10.1109/TCBB.2019.2907492 (2019).

37. Godehard, S.P., Badenhorst, C.P.S., Müller, H., Bornscheuer, U.T. Protein Engineering for Enhanced Acyltransferase Activity, Substrate Scope, and Selectivity of the Mycobacterium smegmatis Acyltransferase MsAcT. ACS Catal. 10, 7552-7562 (2020).

38. Land, H., Hendil-Forssell, P., Martinelle, M. Berglund, P. One-pot biocatalytic amine transaminase/acyl transferase cascade for aqueous formation of amides from aldehydes or ketones. Catal. Sci. Technol., 6, 2897-2900 (2016).

\section{Acknowledgements}

This project was supported by the European Union's Horizon 2020 research and innovation programme under the Marie Skłodowska-Curie grant agreement no. 792804 AROMAs-FLOW (M. L. C.), and SNSF (200021_192274, F. P.). The authors wish to thank Prof. Philippe Renaud (DCB, University of Bern) for insightful scientific discussions.

\section{Author Contributions}


F.P. developed the original concept. M.L.C. performed the experimental work and analysed the results. D.R.P. conceived and performed the computational experiments. M.L.C., F.M. and F.P. conceived and designed the experiments. All authors co-wrote the manuscript.

\section{Competing interests}

The authors declare no competing interests.

Table 1. S-acetylation of different thiols with vinyl acetate as acetyl donor

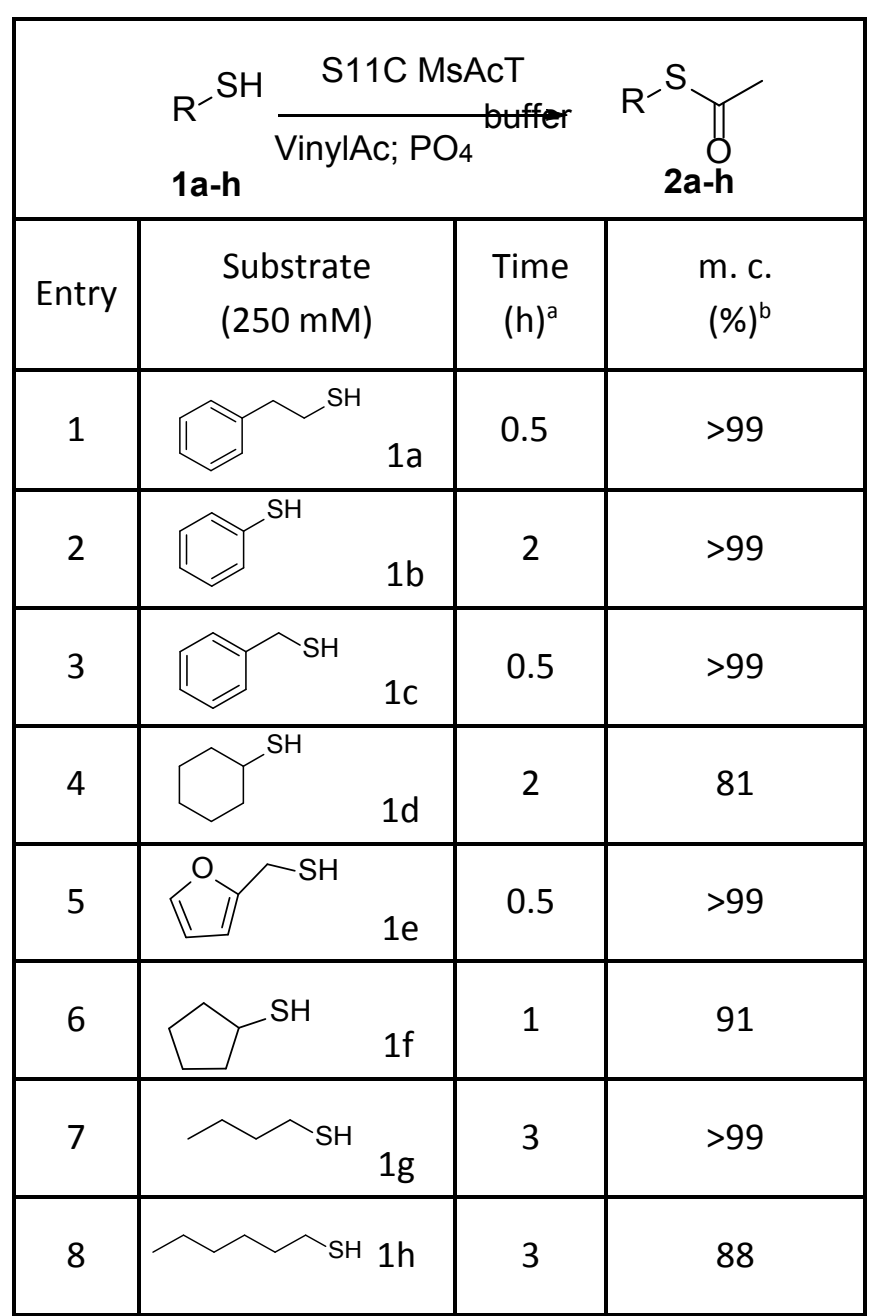

Reactions were performed in the presence of $250 \mathrm{mM}$ substrate and vinyl acetate $10 \% \mathrm{v} / \mathrm{v}$ at $25{ }^{\circ} \mathrm{C}$ in phosphate buffer (100 mM, pH 8.0). $1 \mathrm{mg} / \mathrm{mL}$ enzyme was used. ${ }^{a}$ Time corresponding to maximum conversion. ${ }^{b}$ Determined by GC. $\mathrm{m} . \mathrm{c} .=$ molar conversion. For analytical details see Supplementary Methods. 
Table 2. Screening of different acyl donor

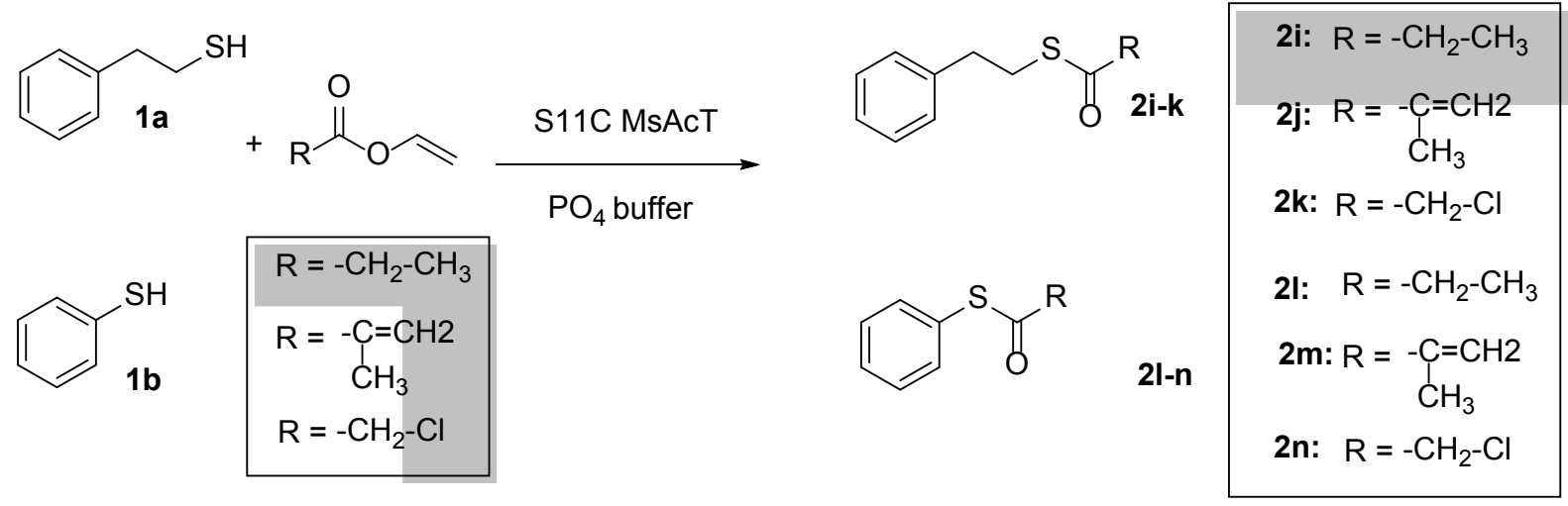

\begin{tabular}{|c|c|c|c|c|c|c|c|}
\hline Entry & $\begin{array}{c}\text { Substrate } \\
(250 \mathrm{mM})\end{array}$ & $\begin{array}{c}\text { Time } \\
(\mathrm{h})^{\mathrm{a}}\end{array}$ & $\begin{array}{c}\text { Vinyl propionate } \\
\text { m. c. }(\%)^{\mathrm{b}}\end{array}$ & $\begin{array}{c}\text { Time } \\
(\mathrm{h})^{\mathrm{a}}\end{array}$ & $\begin{array}{c}\text { Vinyl methacrylate } \\
\text { m. c. (\%) }\end{array}$ & $\begin{array}{c}\text { Time } \\
(\mathrm{h})^{\mathrm{a}}\end{array}$ & $\begin{array}{c}\text { Vinyl chloroacetate } \\
\text { m. c. (\%) }\end{array}$ \\
\hline 1 & $1 \mathrm{a}$ & 3 & $>99$ & 6 & 70 & 5 & 80 \\
\hline 2 & $1 \mathrm{~b}$ & 2 & $>99$ & 5 & $93 \%$ & 2 & $88 \%$ \\
\hline
\end{tabular}

Reactions were performed in the presence of $250 \mathrm{mM}$ substrate and acyl donor $10 \% \mathrm{v} / \mathrm{v}$ at $25^{\circ} \mathrm{C}$ in phosphate buffer (100 mM, pH 8.0). $1 \mathrm{mg} / \mathrm{mL}$ enzyme was used. a Time corresponding to maximum conversion. ${ }^{\mathrm{b}}$ Determined by GC. For analytical details see Supplementary Methods. m. c. = molar conversion.

Table 3. $\mathrm{N}$-acetylation of different secondary amines with vinyl acetate

\begin{tabular}{|c|c|c|c|}
\hline $\mathrm{R}^{-\mathrm{H}_{-\mathrm{R}^{\prime}}^{\mathrm{H}}}$ & $\mathrm{R}^{\prime} \frac{\mathrm{S} 11 \mathrm{C} \mathrm{MsAc}}{\text { VinylAc; } \mathrm{PO}_{4}}$ & & \\
\hline Entry & $\begin{array}{c}\text { Secondary amine } \\
(250 \mathrm{mM})\end{array}$ & $\begin{array}{r}\text { Time } \\
(\mathrm{h})^{\mathrm{a}}\end{array}$ & $\begin{array}{l}\text { m.c. } \\
(\%)^{b}\end{array}$ \\
\hline 1 & 3a & 5 & 65 \\
\hline 2 & $3 h$ & 2 & $>99$ \\
\hline 3 & $3 c$ & 24 & 30 \\
\hline 4 & $3 d$ & 48 & - \\
\hline 5 & Зе & 24 & $>99$ \\
\hline
\end{tabular}




\begin{tabular}{|c|cc|c|c|}
\hline 6 & \multicolumn{2}{|c|}{$3 f$} & 24 & $>99$ \\
\hline 7 & & 3 & 90 \\
\hline
\end{tabular}

Reactions were performed in the presence of $250 \mathrm{mM}$ substrate and vinyl acetate $10 \% \mathrm{v} / \mathrm{v}$ at $25^{\circ} \mathrm{C}$ in phosphate buffer (100 mM, pH 8.0). $1 \mathrm{mg} / \mathrm{mL}$ enzyme was used. ${ }^{a}$ Time corresponding to maximum conversion. ${ }^{\mathrm{b}}$ Determined by HPLC or GC. For analytical details see Supplementary Methods. m. c. = molar conversion.

Table 4. Enantioselectivity in acetylation reaction with vinyl acetate

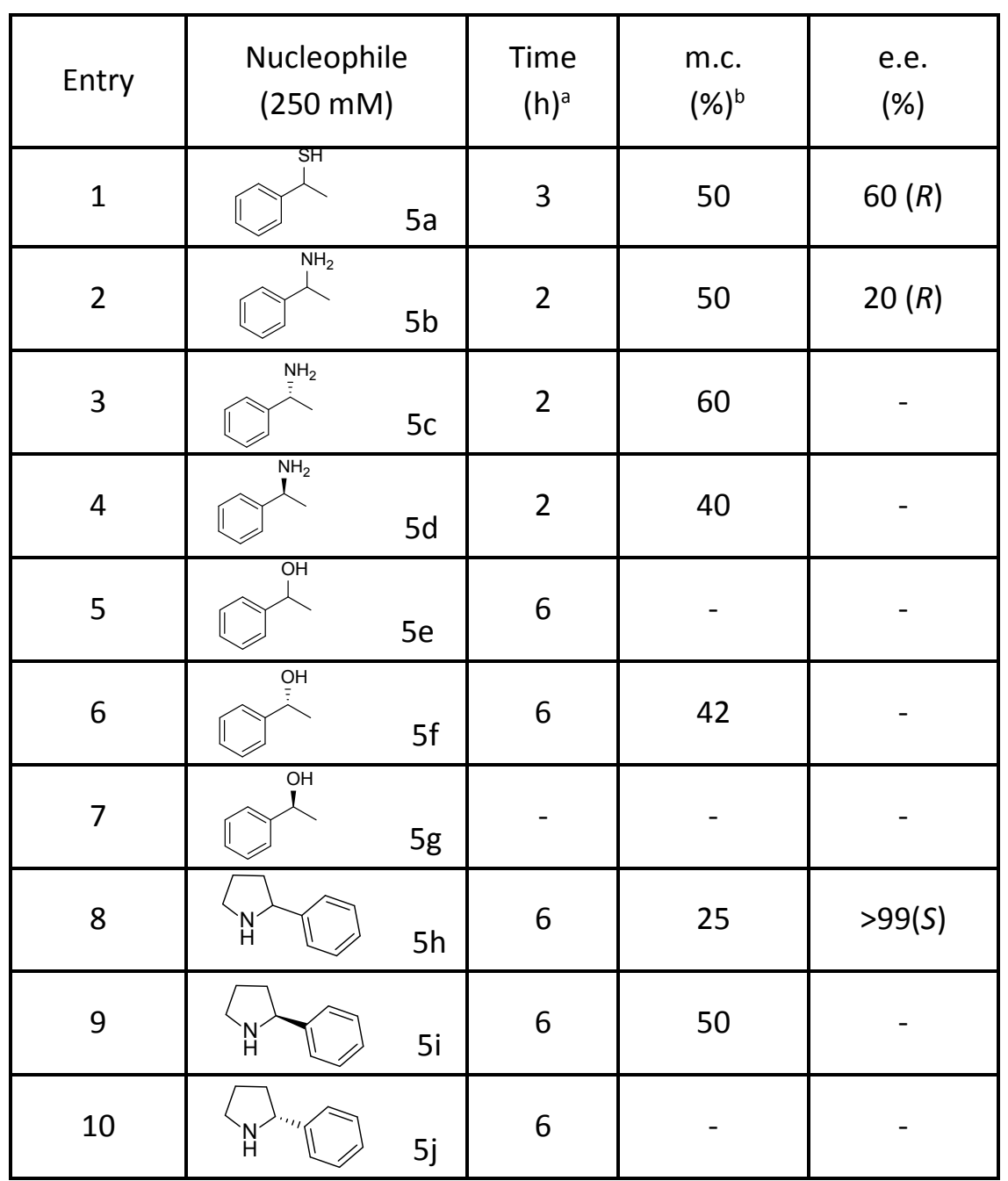

Reactions were performed in the presence of $250 \mathrm{mM}$ substrate and vinyl acetate $10 \% \mathrm{v} / \mathrm{v}$ at $25{ }^{\circ} \mathrm{C}$ in phosphate buffer (100 mM, pH 8.0). $1 \mathrm{mg} / \mathrm{mL}$ enzyme was used. a Time corresponding to maximum conversion. ${ }^{b}$ Determined by HPLC or GC. For analytical details see. Supplementary Methods. m. c. = molar conversion. e.e. $=$ enantiomeric excess. 
Figure 1. Biosynthesis of acetyl-CoA and its mimetics

Reactions were performed in the presence of $250 \mathrm{mM}$ substrate or $100 \mathrm{mM}$ CoA and vinyl acetate $10 \% \mathrm{v} / \mathrm{v}$ at $25^{\circ} \mathrm{C}$ in phosphate buffer $(100 \mathrm{mM}, \mathrm{pH} 8.0) .1 \mathrm{mg} / \mathrm{mL}$ enzyme was used.

\section{Figure 2. Docking of acetyl-CoA}

Details of the final product, acetyl-CoA (yellow) docked in a catalytic position. The grey cartoon indicates the different chains of S11C MsAcT. Its catalytic triad is shown in red and bold letters while the oxyanion hole residues indicated in italics. Hydrogen bonds are depicted in light blue.

\section{Figure 3. CoA and acetyl-CoA computational studies}

A. Representation of the originally identified channel (red) and the lengthened channel (blue). B. Energy profile of CoA travelling to the catalytic site. The colours for the tunnels are maintained from $A$ and the possible catalytic conformations are marked with a black circle. C. Possible catalytic poses of CoA and acetyl-CoA.. 